\title{
Investigating thermal properties of gas-filled planetary regoliths using a thermal probe
}

\author{
M. D. Paton ${ }^{1}$, A.-M. Harri ${ }^{1}$, T. Mäkinen ${ }^{1}$, and S. F. Green ${ }^{2}$ \\ ${ }^{1}$ Finnish Meteorological Institute, P.O. Box 503, Helsinki, Finland \\ ${ }^{2}$ PSSRI, The Open University, Milton Keynes, MK7 6AA, UK \\ Correspondence to: M. D. Paton (mark.paton@ @mi.fi)
}

Received: 22 August 2011 - Published in Geosci. Instrum. Method. Data Syst. Discuss.: 22 December 2011

Revised: 23 February 2012 - Accepted: 6 March 2012 - Published: 29 March 2012

\begin{abstract}
We introduce a general purpose penetrator, fitted with a heater, for measuring temperature and thermal diffusivity. Due to its simplicity of deployment and operation the penetrator is well suited for remote deployment by spacecraft into a planetary regolith. Thermal measurements in planetary regoliths are required to determine the surface energy balance and to measure their thermal properties. If the regolith is on a planet with an atmosphere a good understanding of the role of convection is required to properly interpret the measurements. This could also help to identify the significant heat and mass exchange mechanisms between the regolith and the atmosphere. To understand the role of convection in our regolith analogues we use a network of temperature sensors placed in the target. In practical applications a penetrator will push material out of the way as it enters a target possible changing its thermal properties. To investigate this effect a custom built test rig, that precisely controls and monitors the motion of the penetrator, is used. The thermal diffusivity of limestone powder and sand is derived by fitting a numerical thermal model to the temperature measurements.

Convection seems to play an important role in the transfer of heat in this case. Firstly a diffusion-convection model fits the laboratory data better than a diffusivity-only model. Also the diffusivity derived from a diffusivity-convection model was found to be in good agreement with diffusivity derived using other methods published in the literature. Thermal diffusivity measurements, inspection of the horizontal temperature profiles and visual observations suggests that limestone powder is compacted more readily than sand during entry of the penetrator into the target. For both regolith analogues the disturbance of material around the penetrator was determined to have an insignificant effect on the diffusivity measurements in this case.
\end{abstract}

\section{Introduction}

A convenient method of measuring thermal properties of particulate materials, in-situ, is by using a probe that penetrates the target (Banaszkiewicz et al., 2007). The probe consists of a heating element coupled with a temperature sensor situated half way along its length. It is characteristically long and thin and so can be easily driven into a sample for making measurements. The dimensions are dictated by theoretical considerations. An infinitely long cylinder, which the probe approximates, can be thermally modelled using an analytical solution to the heat-conduction equation. The hot wire method is similar to the probe method except that the heating element doubles up as a heat sensor. The wire is not stiff and it is best suited to measuring thermal conductivity in gases and liquids (Birchley et al., 1992).

A method similar to hot-wire and probe methods, but based on a different geometry, is the spherical heat source. Due to its small size it is best suited to liquids and consolidated materials (van Gelder, 1998). All these methods use an active heat source.

Thermal properties, such as thermal conductivity and thermal inertia can be measured by monitoring the temperature of a probe as it attains thermal equilibrium with its surrounding. Such measurements can be made using the Bullard probe (Bullard, 1954).

The Bullard probe was primarily designed to measure the heat flow through the sea floor. It consisted of a long thin cylinder which penetrated the sea floor and was fitted with temperature sensors, one fitted at the top and one at the bottom. Weights and recording equipment were mounted above the penetrating part of the probe. The temperature difference between the sensor gives the temperature gradient. The 
thermal conductivity can be obtained from laboratory measurements of cored samples, to be used together with the temperature gradient, to calculate the heat flow. Bullard was also able to infer thermal properties of the marine sediments, in situ, by analysing the passive cooling of the probe after penetrating the sea floor.

Penetrators are devices that penetrate the ground using a variety of mechanisms and with a wide range of speeds (centimetres per second to $100 \mathrm{~s}$ of metres per second). In planetary exploration high-speed penetrators have been used as part of an anchoring system (Stöcker and Thiel, 1998) or ballistic delivery of science instruments into the subsurface or on the surface (Smrekar et al., 1999; Harri et al., 2003). Low-speed penetrators are normally deployed from a landed spacecraft as with the hammer-driven MUPUS probe (Spohn et al., 2007) or Beagle 2 mole (Pinna et al., 2001).

Penetrators generally consist of a hollow slender cylindrical shaft with a shaped tip mounted on the penetrating end. Internal temperature sensors can be easily fitted into penetrators as they are small, robust and simple to use. For example the Deep Space 2 measurements used the rate of cooling to determine thermal properties of the surrounding Martian regolith. If the thermal properties of the penetrator are known then the thermal properties of the surrounding material can be determined (Urquhart and and Smrekar, 2000; Paton et al., 2010). Numerical models are required to solve the heat transfer equation as a penetrator will be far from the idealised geometry and the homogenous composition that is a requirement for the application of an analytical solution.

An example of a penetrator specifically designed to make thermal measurements is the MUPUS thermal probe. MUPUS is part of the Rosetta lander, Philae's experiment suite to investigate the thermal and mechanical behaviour of the outer layers of a comet (Spohn et al., 2007). The thermal probe is a hammer driven penetrator that has temperature sensors mounted on the inside of its hollow shaft and may penetrate to a depth of $0.35 \mathrm{~m}$. The resistance of the sensors are a function of temperature and is well known. The sensors can be used in passive mode to determine the temperature gradient with depth or in active mode, where the sensors act also as heaters, to determine the thermal conductivity depth profile.

Penetrometers are a specialised type of ground penetrating instruments, used to make mechanical measurements with a force sensor, normally mounted behind the tip (Lunne et al., 1997; Rowe, 2001). They are deployed from a platform sitting on the surface that can supply power for a deployment mechanism such as compressed air for pneumatics or electricity for operating a hammer. Penetrometer speeds are normally of the order of centimetres per second.

With low speed penetrators and penetrometers the frictional forces and heating will be low with the possibility of mounting sensors on the outside surface of the shaft. One possibility is to determine the thermal properties of the surrounding material using a dual purpose film sensor-heater de- vice wrapped around the shaft. Similar flexible dual sensorheater devices have been used before on the MUPUS thermal probe. This type of sensor allows direct contact with the target material. For making thermal property measurements this is particularly useful, because it is easier to thermally isolate a sensor or heater from the penetrator body, when it is mounted on the outside. If the heater-sensor is not thermally isolated from the penetrator and if the penetrator body has a higher conductivity than the surrounding material, the majority of the heat will be quickly carried away and spread into the penetrator.

Knowing the thermal properties of the penetrator construction materials and the dimensions of its structure is required as a thermal probe will influence the temperature field around it. A numerical model can be used to invert the data (Hagermann and Spohn, 1999) if the thermal properties of the penetrator are known. If such a model is applied to the measured temperature to derive the thermal properties as in Paton et al. (2010), and the heater-sensor is not thermally isolated from the penetrator, then an uncertainty in knowing the penetrator thermal properties will be transferred to the derived thermal properties of the target material.

A hollow device is preferable, especially for extraterrestrial applications, because of the potential mass savings and the possibility of housing extra instruments for further subsurface explorations. If the probe is hollow and used in an environment with a significant atmosphere then convection inside the penetrator could be observed (Kömle et al., 2002). This could also add an uncertainty to the derived thermal properties of the target material.

This paper is structured as follows. First, heat transfer in porous planetary regoliths is discussed. Then the formulation of the problem of heat transfer in such materials is outlined. The penetrator is introduced and a numerical solution to the equations is proposed. An experiment is described that uses a test rig to conduct impact tests on the target and temperature observations inside the target to look for evidence of convective flow within the targets. We then present experimental results and fits of the model output to the data. The model is discussed in terms of the convection in the media and the presence of a compaction zone around the penetrator. Recommendations are made for the improvement of the experimental design. The design of the penetrator is assessed and design recommendations are made for its use on other Solar System bodies.

\section{Heat transfer in porous planetary regoliths}

There are a number of thermal exchange processes and related material properties that may contribute to the heat transfer through a given material. The most important processes controlling heat transfer are due to thermal conduction, thermal radiation, advection and evaporation of volatiles. Thermal conduction will depend on the material composition and its micro-structure (i.e. the contact area between separate 
elements that make up the bulk material, for example, the contact area between grains of sand). The micro-structure of a granular material will partly depend on the microstructural properties of the grains such as size and the surface texture. Heat transfer by radiation will depend on the temperature gradient within the material. Natural convection will be dependent on the temperature gradient in the material coupled together with the thermal properties of the fluid flowing through the material. Advection (e.g. winds) is similarly dependent on the thermal properties of the fluid and also its velocity. Evaporation and condensation of volatiles will transfer heat to and from a material due to the exchange of latent heat to and from the volatile material (Incropera and Dewitt, 2002; Harri et al., 1998).

Extraterrestrial surfaces are often found to be covered in a blanket of particles, called a regolith (Scott and Pain, 2009). Examples of bodies that have regoliths are Mercury, the Moon, Mars, Titan and asteroids. On airless bodies, like Mercury and the Moon, the regolith is generated from asteroid and meteorite sized objects impacting solid bedrock, breaking it up to produce fragments varying in size from particles less than $1 \mathrm{~mm}$ in size to rocks and to boulders. Then a steady stream of micrometeorite and charged particle impacts break down the larger impact fragments, over billions of years, into a layer of fine dust on top of the coarser material remaining underneath. Further impacts into the regolith, by the larger impactors, will mix the top layers producing a poorly sorted regolith. On Mars a similar process occurs except erosion by micromeorite and charged particle bombardment is prevented by the Martian atmosphere. Particles in the Martian regolith are thought to evolve, due to aelion process, into smaller dust size particles that are then transported all around the planet by the winds.

Due to its particulate nature the regolith is porous with a large fraction of particle surface exposed to the gases, and perhaps liquids, filling the voids between them. On Earth the thermal properties of the regolith (or more strictly soil when plants are present) are modified by the presence of large quantities of liquid water. For example, the thermal conductivity of dry sand changes by an order of magnitude when saturated with liquid water (Smits et al., 2009). Similarly water ice content can vary the thermal conductivity by orders of magnitude. On Mars water ice may be present in the regolith as layers below the surface as found by the Phoenix lander (Smith et al., 2009). It may also be deposited on the surface as a frost as observed by the Phoenix and Viking 2 landers. On Mars the thermal properties of the regolith could then change noticeable over seasonal and daily time periods due to the exchange of volatiles between the regolith and the atmosphere (Svitek and Murray, 1990; Harri et al., 1999).

The thermal conductivity of the regolith will change due to the change in its bulk composition (Gori and Corasaniti, 2004). Also, on the micro-scale, the thermal conductivity will depend on the contact area between the grains which may change as the ice sublimates from between the grains.
The thermal conductivity of a composite material, like a dustice regolith mixture, is called the effective conductivity. The atmosphere will penetrate into the pores of the regolith and may also play an important role in controlling the effective conductivity of the regolith.

The effective thermal conductivity of monodisperse glass bead samples was measured by Hütter et al. (2008) in air at a range of pressures from 1 atmosphere to $10^{-9}$ atmosphere. The effective thermal conductivity was found to decrease with decreasing atmospheric pressure for all samples. At low pressures the thermal conductivity was found to be dependent on the particle size with the thermal conductivity increasing with particle size. Similar measurements were made by Presley and Christensen (1997) using glass beads but in a carbon dioxide atmosphere. Both authors found that the effective thermal conductivity of glass beads was particularly sensitive to pressure changes in the range of pressures found in the Martian atmosphere.

Radiative heat transfer between particles in the regolith can increase the effective thermal conduction relative to the other mechanisms when the temperatures are high enough because it scales with the temperature cubed. Hence radiation is required in thermal models to accurately describe the thermal behaviour of the regolith, especially of airless bodies such as Mercury and the Moon (e.g. Ulrichs and Campbell, 1969).

\section{Formulation of the problem}

To calculate the temperature of a particulate material, with gas penetrating between the pores, the flow rate of this gas component needs to be determined. This information can then be used to calculate the heat transfer across the solid component of the material and the temperature field of the bulk material. This section introduces the relevant parameters and then formulates the physics of this problem based on these parameters.

\subsection{Principal physical parameters}

There are a number of parameters that will control the flow of the fluid through a porous medium, the most important being porosity, permeability and dynamic viscosity. Heat transfer in porous granular material is also sensitive to the microstructural parameters of porosity and the Hertz factor.

The viscosity relates the shearing forces in a moving fluid to the velocity gradient. A velocity gradient will develop in a slow-moving fluid close to a boundary such as a wall. The faster the flow the larger the velocity gradient, with larger shearing forces are produced. The shearing stress between layers in a laminar flow can be written as $\tau=\mu \partial u / \partial y$ where $\mu$ is the dynamic viscosity, $u$ is the velocity of the fluid at a distance, $y$ from a wall. Viscosity is dependent on temperature. For air the dynamic viscosity increases with 
increasing temperature from $1.98 \times 10^{-5} \mathrm{Pas}$ at $300 \mathrm{~K}$ to $2.29 \times 10^{-5}$ Pa s at $400 \mathrm{~K}$.

The permeability is expressed as $K=v \mu \Delta x / \Delta P$ where $v$ is the superficial velocity (or volumetric flux), $\mu$ is the dynamic viscosity, $\Delta x$ is the thickness of the bed of the porous medium and $\Delta P$ is the applied pressure difference. The permeability of sand varies from $10^{-9}$ to $10^{-12} \mathrm{~m}^{2}$.

The porosity describes the ratio of the void volume to solid volume of a medium. For example sand consists of an assembly of grains that have empty spaces between them. The grains themselves may also have a small amount of porosity due to internal fractures. The porosity can be expressed as $\phi=V / V_{\mathrm{M}}$, where $V$ is the volume of the voids in the medium and $V_{M}$ is the volume of the medium as a whole. The porosity of sand varies between 0.37 and 0.5 . The permeability is a measure of a material's ability to transport a fluid. It is used in geology to describe the characteristics of materials such as rocks and deposited materials such as clays, sands and gravels.

The Hertz factor describes how well objects are in contact with each other. This is an important parameter for modelling of heat transfer by conduction between grains in granular material. It is defined as the ratio of the contact area to the cross-sectional area of the grain $h=A_{\mathrm{c}} / A_{\mathrm{g}}$ where $A_{\mathrm{c}}$ is the area in contact and $A_{\mathrm{g}}$ is the cross-sectional area.

An important thermal parameter is the thermal diffusivity which is the ratio of the thermal conductivity to the volumetric heat capacity and is defined as $\alpha=k / \rho c$ where $k$ is the thermal conductivity, $\rho$ is the density and $c$ is the specific heat capacity. The thermal diffusivity relates the temporal and spatial aspects of heat transfer. It reflects how quickly the temperature at one point will change when the temperature changes at another point.

\subsection{Fluid flow and heat transfer in a porous medium}

Free fluid flow is already complicated and introducing a porous material into the flow increases the complexity of the system. Attempts have been made to obtain an equation equivalent to the Navier-Stokes equation. Several text books and papers present such a generalised equation. For example a general form of fluid flow in porous material can be found in Hsu and Cheng (1990).

$\rho_{\mathrm{f}}\left[\frac{1}{\varphi} \frac{\partial v}{\partial t}+\frac{1}{\varphi} \nabla\left(\frac{v \cdot v}{\varphi}\right)\right]=\nabla P-\frac{\mu}{K} v+\frac{\mu}{\varphi \rho_{\mathrm{f}}} \nabla^{2} v-\frac{C_{\mathrm{F}} \rho_{\mathrm{f}}}{K^{1 / 2}}|v| v$

where $v$ is the velocity vector of the fluid, $\rho_{\mathrm{f}}$ is the fluid density, $P$ is the pressure, $t$ is the time, $v$ is the velocity, $\phi$ is the porosity, $K$ is the permeability and $c_{\mathrm{F}}$ is a dimensionless form-drag constant. At low Reynolds numbers the advection term in the left hand term of equation 1 may be dropped as in porous material the fluid will find it difficult to retain its momentum (Beck, 1972). The last two terms in Eq. (1) describe the drag forces generated by the flow of a fluid through a medium. The first term (i.e. second from last) was introduced by Brinkmann (1947) to describe the macroscopic shearing forces in the bulk material. The second describes the microscopic inertial drag that becomes significant at high Reynolds numbers introduced by Foreheimer (1901). The Brinkmann term can be considered negligible as the scale length of the viscous boundary layer is predicted, in most cases, to be smaller than the particle size. At low Reynolds numbers the Foreheimer term can also be neglected. So for most practical applications the following equation is appropriate for describing fluid flow through porous materials (Nield and Adrian, 2006).

$\rho_{\mathrm{f}} \frac{1}{\varphi} \frac{\partial v}{\partial t}=-\nabla P-\frac{\mu}{K} v$

Equation (1) contains a term on the left due to the pressure field and the microscopic shearing forces (Stoke's law) on the local scale. Equation (2) is essentially a form of Darcy's law that was developed from observations of the flow of water through sand by Darcy (1856). It is applicable for Reynolds numbers below unity, but has been shown to hold up to Reynolds numbers of 10 , as this is a transition zone between the significance of viscous and inertial forces (e.g. Andrade Jr. et al., 1999).

Darcy's law has been derived theoretically from the Navier-Stokes equation by several authors (Neuman, 1977) and correctly describes the behaviour of fluids in porous materials in experiments (Nield and Adrian, 2006). However the behaviour of gas flows in porous media may differ from those of liquids when compressibility effects become important. This will occur at low pressures or in porous media with small characteristic pore sizes. A correction factor to Darcy's law was introduced by Klinkenberg (1941) and can be used for Knudsen number of 0.01 to 0.1 . Darcy's law can be used to model natural convection processes adequately in porous media for Darcy numbers lower than $\sim 0.0001$ e.g. see Nouri-Borujerdi et al. (2008).

The continuity equation for a fluid in a porous material can be defined in a similar manner as for an incompressible fluid as follows (Nield and Adrian, 2006).

$\varphi \frac{\partial \rho_{\mathrm{f}}}{\partial t}+\nabla\left(\rho_{\mathrm{f}} v\right)=0$

where

$(\rho c)_{\mathrm{m}}=(1-\varphi)(\rho c)_{\mathrm{s}}+\varphi(\rho c)_{\mathrm{f}}$

$k_{\mathrm{m}}=(1-\varphi) h k_{\mathrm{s}}+\varphi k_{\mathrm{f}}$

where $k_{\mathrm{m}}$ is the thermal conductivity of the bulk material, $k_{\mathrm{s}}$ is the thermal conductivity of solid component, $k_{\mathrm{f}}$ is the thermal conductivity of the fluid component, $(\rho c)_{\mathrm{m}}$ is the volumetric heat capacity of the bulk material, $(\rho c)_{\mathrm{S}}$ is the volumetric heat capacity of the solid component and $(\rho c)_{\mathrm{f}}$ is the volumetric heat capacity of the fluid component. 


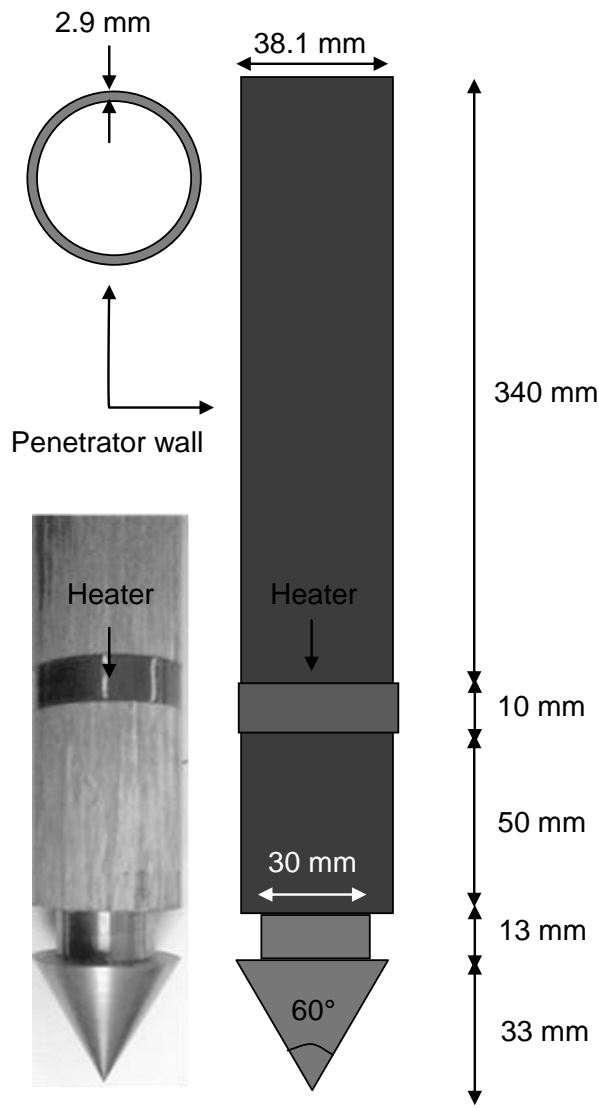

Fig. 1. A photograph (left) and schematic diagram (right) of the penetrator and thermal sensor.

\section{Application of the numerical thermal model}

A penetrator fitted with a thermal sensor, shown in Fig. 1, was designed and built in-house for making thermal property measurements in planetary regoliths. The penetrator geometry and dimensions follow the standard penetrometer type used in the Cone Penetration Test (CPT). The reason for doing this was that the penetrator was developed in tandem with a penetrometer (Paton, 2005) and a standard reference was required. The intention was to eventually merge the designs into one device that could conduct both penetrometry and then continue, once deployed, with thermal measurements. In this way a mechanical and thermal measurements could be used to derive the material physical properties using two independent methods. Also the wealth of CPT measurements available could assist with the interpretation of the penetrometer measurements.

The shaft of the penetrator is a glass reinforced plastic pultrusion. Pultrusion is a manufacturing process for extended shapes that have a constant cross-section. The material is pulled through a shaping die to achieve this. A material produced this way might have anistropic thermal properties because of the production process. It was chosen because it is both strong and has a low thermal conductivity. A shaft with low thermal conductivity perturbs the heat transfer characteristics of the surrounding material less than a shaft using a highly conductive material such as steel. A foil heater was wrapped around the shaft of the penetrator to make active heat measurements. The heater doubles as a heater and a temperature sensor. The heater used was a Minco thermo foil heater model 5228, which is also used as a temperature sensor. It has a length of $100 \mathrm{~mm}$, a height of $10 \mathrm{~mm}$ and a thickness of $0.3 \mathrm{~mm}$. The element is made of nickel and increases in resistance with temperature. The element is embedded in Kapton, which is a good insulator. The resistance of the heater at $0^{\circ} \mathrm{C}$ is $238 \mathrm{ohms}$.

The foil heater is shown in Fig. 1 wrapped around the shaft of the penetrator. The foil heater was chosen because its resistance changes little with temperature and so a constant voltage source can be used to provide an approximately constant power output over a wide range of foil temperatures. The foil heater was placed near the tip to ensure measurements were made deep enough to avoid boundary effects from the material's surface. A dummy load cell, made of steel, was inserted behind the penetrator tip, to approximately reproduce the thermal properties of a real load cell as used in Paton (2005). A steel tip was used as thermal modelling indicated that the heater was suitably placed to avoid any influence from the tip. The thermal properties of the tip and other parts of the penetrator are listed in Table 1.

Using a discrete form of the heat equation allows a thermal model of a penetrator to be constructed as discrete control volumes (or elements). Each control volume has its own thermal properties, for example, conductivity, density, heat capacity and heat production. These control volumes are then coupled to neighbouring control volumes using the heat equation in its discrete form. The heat equation, in the 2-D cylindrical coordinates of radial distance and depth (appropriate for a cylindrically symmetric penetrator entering a porous medium vertically), is as follows.

$(\rho c)_{\mathrm{m}} \frac{\partial T}{\partial t}=\frac{1}{r} \frac{\partial}{\partial r}\left(k_{\mathrm{m}} r \frac{\partial T}{\partial r}\right)+\frac{\partial}{\partial z}\left(k_{\mathrm{m}} \frac{\partial T}{\partial z}\right)+v_{z}(\rho c)_{\mathrm{f}} \frac{\partial T}{\partial z}+S$

where $r$ is the radial distance, $z$ is the vertical distance into the material, $T$ is the temperature, $t$ is the time, $v_{z}$ is the vertical speed of the fluid and $S$ is the volumetric heat production. The momentum equation describing fluid flow in the vertical coordinate is as follows.

$\frac{\partial v_{z}}{\partial t}=-\frac{\varphi}{\rho_{\mathrm{f}}}\left(\frac{\partial P}{\partial z}+\frac{\mu}{K} v_{z}\right)+\varphi g$

where $g$ is the acceleration due to gravity. We only consider fluid flow in the vertical direction as we are dealing with natural convection which is assumed to be dependent on the vertical pressure gradient only.

A grid of control volumes is visualised in Fig. 2. The discrete form of the heat equation is obtained by integrating it over a control volume following the method outlined by 
Table 1. Thermal properties of the penetrator components.

\begin{tabular}{llccc}
\hline Penetrator component & Material & $\begin{array}{c}\text { Thermal conductivity } \\
\left(\mathrm{W} \mathrm{m}^{-1} \mathrm{~K}^{-1}\right)\end{array}$ & $\begin{array}{c}\text { Density } \\
\left(\mathrm{kg} \mathrm{m}^{-3}\right)\end{array}$ & $\begin{array}{c}\text { Heat capacity } \\
\left(\mathrm{J} \mathrm{kg}^{-1} \mathrm{~K}^{-1}\right)\end{array}$ \\
\hline Shaft & Fibreglass & 0.370 & 1800 & 1554 \\
Sensor insulation & Kapton & 0.225 & 1042 & 1090 \\
Sensor element & Nickel & 91.73 & 8900 & 461 \\
Sensor adhesive & Sticky tape & 0.108 & 512 & 1255 \\
Dummy load cell & Stainless steel & 15.10 & 8055 & 480 \\
Tip & Stainless steel & 15.10 & 8055 & 480 \\
\hline
\end{tabular}

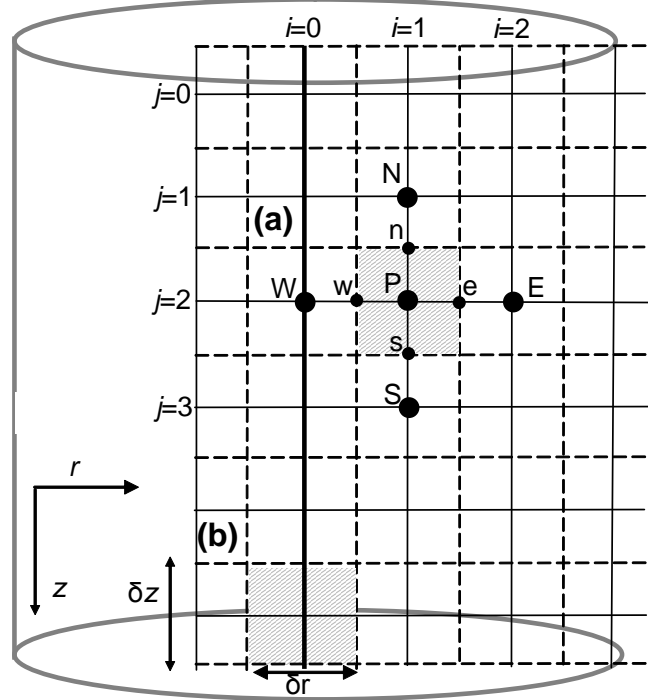

Fig. 2. Grid structure and notation describing control volumes used for numerical thermal modelling of a penetrator fitted with a thermal sensor in analogue planetary regoliths.

Patankar (1980). The centres of control volumes are located at the point where the solid lines cross. The control volume location is referenced with increments of one starting at zero on both the $z$ and $r$ axis. Figure 2 shows control volume ( $a$ ) with its centre labelled with the letter $\mathrm{P}$ (pole). The centres of neighbouring control volumes to the left and right are labelled with the capital letters, W (west), E (east), N (north) and $S$ (south). The temperature of the control volume is then referred to as $T_{\mathrm{P}}$. The upper, right, lower and left boundaries of the control volumes are marked with the letters n, e, s and $\mathrm{w}$ respectively. These markers represent the boundaries over which the heat-conduction equation is integrated.

Using the grid system as a coordinate system for the control volumes as shown in Fig. 2 the momentum equation can be written as follows in discrete form and solved for change in vertical velocity as follows.

$\delta v=-\varphi \frac{P_{\mathrm{n}}-P_{\mathrm{S}}}{\left(\rho_{\mathrm{f}}\right)_{\mathrm{P}}} \frac{\delta t}{\delta z}+\mu \frac{v_{\mathrm{N}}-2 v_{\mathrm{P}}+v_{\mathrm{S}}}{\left(\rho_{\mathrm{f}}\right)_{\mathrm{P}}\left(\rho_{\mathrm{f}}\right)_{\mathrm{P}}} \frac{\delta t}{\delta z^{2}}-\frac{\varphi \mu}{K} \frac{v_{\mathrm{P}}}{\left(\rho_{\mathrm{f}}\right)_{\mathrm{P}}} \delta t$
The velocity due to the pressure term will be dependent on the porosity because the surface area will be reduced by the presence of the solid structure. In a similar manner the heat transfer equation can be rewritten and solved for temperature as follows.

$\delta T=a_{\mathrm{e}}\left[T_{\mathrm{E}}-T_{\mathrm{P}}\right]-a_{\mathrm{w}}\left[T_{\mathrm{P}}-T_{\mathrm{W}}\right]+a_{\mathrm{n}}\left[T_{\mathrm{N}}-T_{\mathrm{P}}\right]-a_{\mathrm{s}}\left[T_{\mathrm{P}}-T_{\mathrm{S}}\right]+b$

$a_{\mathrm{e}}=\frac{\left(k_{\mathrm{m}}\right)_{\mathrm{e}}}{\rho_{\mathrm{m}} c_{\mathrm{m}}} \frac{1+0.5 i}{\delta r^{2}} \delta t$

$a_{\mathrm{w}}=\frac{\left(k_{\mathrm{m}}\right)_{\mathrm{w}}}{\rho_{\mathrm{m}} c_{\mathrm{m}}} \frac{1-0.5 i}{\delta r^{2}} \delta t$

$a_{\mathrm{n}}=\frac{\left(k_{\mathrm{m}}\right)_{\mathrm{n}}}{\rho_{\mathrm{m}} c_{\mathrm{m}}} \frac{\delta t}{\delta z^{2}}+v_{\mathrm{n}} \frac{\rho_{\mathrm{f}} c_{\mathrm{f}}}{\rho_{\mathrm{m}} c_{\mathrm{m}}} \frac{\delta t}{\delta z}$

$a_{\mathrm{s}}=\frac{\left(k_{\mathrm{m}}\right)_{\mathrm{s}}}{\rho_{\mathrm{m}} c_{\mathrm{m}}} \frac{\delta t}{\delta z^{2}}+v_{\mathrm{s}} \frac{\rho_{\mathrm{f}} c_{\mathrm{f}}}{\rho_{\mathrm{m}} c_{\mathrm{m}}} \frac{\delta t}{\delta z}$

$b=\frac{s}{\rho_{\mathrm{m}} c_{\mathrm{m}}}$

The conductivity and velocity have the subscripts "n", "e", "s" and " $w$ " that indicate over which boundary the conductivity is to be applied. To calculate the conductivity between two control volumes a harmonic mean is used. This is recommended by e.g. Patankar (1980) and is chosen so that the correct heat flux is modelled for the limiting case where the conductivity goes to zero (for example the interface between a material and a vacuum). An example of a conductivity calculation over a control volume interface is shown in Eq. (14).

$k_{\mathrm{W}}=\frac{2 k_{\mathrm{P}} k_{\mathrm{W}}}{k_{\mathrm{W}}+k_{\mathrm{P}}}$

In deriving Eq. (8) an assumption had to be made over how the temperatures vary with time between time steps. There exist a range of possibilities. As an example we use the case where $T_{t}>T_{t+\delta t}$ where $T$ is the temperature, $t$ is the time and $\delta t$ is the time step. Between times $t$ and $t+\delta t$ the temperature could remain constant and then drop down to temperature $T_{t+\delta t}$ at the last moment (explicit scheme). Alternatively it could decrease in a linear fashion (Crank-Nicholson 


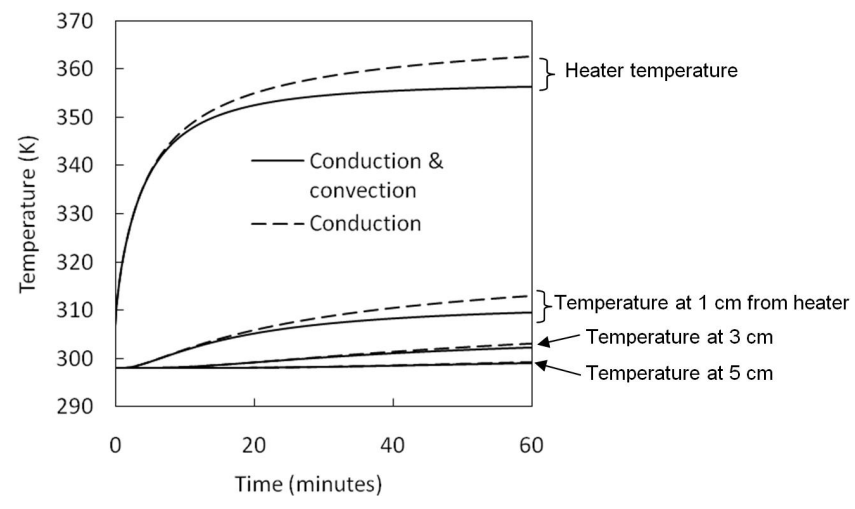

Fig. 3. Comparison of temperatures from models that are identical except that one includes convection in the target (solid lines) and the other is without convection in the target (dotted lines). In both cases convection is modelled inside the hollow shaft of the penetrator.

scheme) or it could drop down instantly to the lower temperature (implicit scheme). The implicit scheme approximates more closely the exponential decay profile intrinsic to temperature-time relationships for heat transfer by diffusion. Also the implicit scheme will not produce unphysical results to mathematical instabilities inherent in the explicit and Crank-Nicholson schemes.

For convection for all cases it is physically unrealistic if the fluid particle is moving so fast that it travels across a control volume in a time shorter than the model time step. Therefore there is no advantage using an implicit scheme over an explicit scheme in terms of stability (Fletcher, 1988). CrankNicholson and Implicit schemes are more accurate at small time steps but we are interested in using as large time steps as possible to make efficient use of computer resources. We apply an explicit scheme to Eqs. (7) and (8) which can then be written as.

$$
\begin{aligned}
v_{\mathrm{P}}^{1} & =-\varphi \frac{P_{\mathrm{n}}^{0}-P_{\mathrm{s}}^{0}}{\left(\rho_{\mathrm{f}}\right)_{\mathrm{P}}^{0}} \frac{\delta t}{\delta z}+\mu \frac{v_{\mathrm{N}}^{0}-2 v_{\mathrm{P}}^{0}+v_{\mathrm{s}}^{0}}{\left(\rho_{\mathrm{f}}\right)_{\mathrm{P}}^{0}\left(\rho_{\mathrm{f}}\right)_{\mathrm{P}}^{0}} \frac{\delta t}{\delta z^{2}} \\
& -\frac{\varphi \mu}{K} \frac{v_{\mathrm{P}}^{0}}{\left(\rho_{\mathrm{f}}\right)_{\mathrm{P}}^{0}} \delta t+v_{\mathrm{P}}^{0} \\
T_{\mathrm{P}}^{1} & =a_{\mathrm{e}}^{0}\left[T_{\mathrm{E}}^{0}-T_{\mathrm{P}}^{0}\right]-a_{\mathrm{w}}^{0}\left[T_{\mathrm{P}}^{0}-T_{\mathrm{W}}^{0}\right]+a_{\mathrm{n}}^{0}\left[T_{\mathrm{N}}^{0}-T_{\mathrm{P}}^{0}\right] \\
& -a_{\mathrm{s}}^{0}\left[T_{\mathrm{P}}^{0}-T_{\mathrm{s}}^{0}\right]+b_{\mathrm{P}}^{0}+T_{\mathrm{P}}^{0}
\end{aligned}
$$

The superscript " 1 " indicates that the temperature is the current temperature to be solved. Superscript " 0 " is attached to a temperature, velocity or density from a previous calculation. Each control volume will be "controlled" with its own equation. It is therefore required that $i \times j$ equations are solved for each time step. Results from the previous iteration are then used in the calculations of the velocity and temperature fields in the present iteration as indicated in Eqs. (15) and (16).

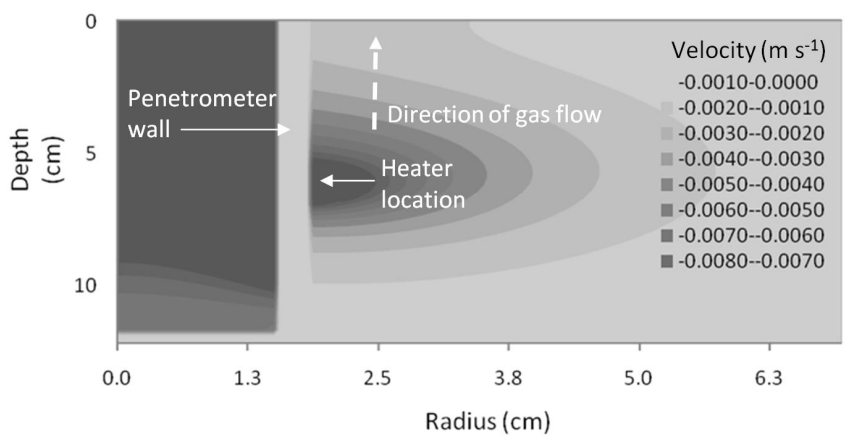

Fig. 4. Velocity field of air in the model of the penetrator and its thermal sensor in sand. The apparent gap is due to the fact that there is no gas in the penetrator wall. To the right of the wall is the target material and to the left is the air inside the penetrator. The contours of the gas velocity inside the penetrator are not resolved as it is off the scale used to show the velocity of the gas in the target material.

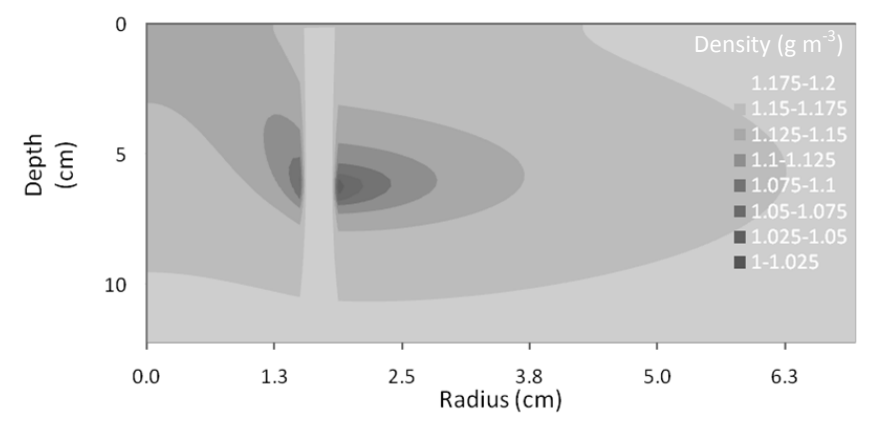

Fig. 5. Density map of air in the model of the penetrator and heater in sand. The gap is due to the fact that the penetrator wall is solid. To the right of the wall is the target material and to the left is the air inside the penetrator.

Shown in Fig. 3 are examples of the numerical model, applied to the penetrator, in a granular material made of rocklike material such as sand. It is clear that convection reduces the temperatures of the material. It is interesting to note that the temperatures appear to diverge after a time of several minutes suggesting in the initial stages natural convection is not so important in the heat transfer. In fact the divergence increases approximately linearly reaching a maximum of $6 \mathrm{~K}$ between the modelled heaters after one hour. This is difficult to see in Fig. 3 in the beginning because of the steep gradient.

Figure 4 shows the velocity of gas in the granular material after $1 \mathrm{~h}$. It is moving very slowly, of the order of centimetres per second within the first few centimetres from the heater. It can be seen from Fig. 3 the convection is obviously stronger inside the penetrator with higher velocities. The plume can be seen more clearly from the density and temperature plots in Figs. 5 and 6, respectively. 


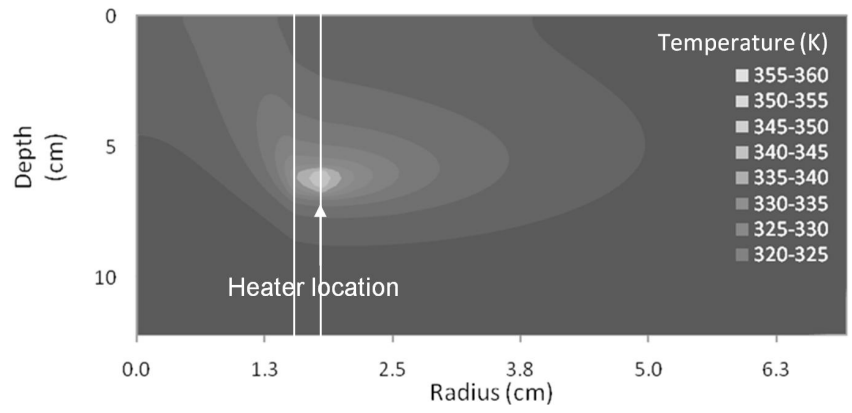

Fig. 6. Temperature map of the air in the model of the penetrator and heater in sand. To the right of the wall is the target material and to the left is the air inside the penetrator.

\section{Experiment design}

The experiment described here was designed essentially to resolve issues relating to the practical application of the penetrator in the field. When the penetrator enters the target it will create a zone of disturbed material whose thermal properties may then be unrepresentative of the undisturbed material thermal properties. To investigate this in a controlled manner a custom built drop rig was used. The rig was designed such that speed at impact could be controlled and measured. Also the location of the penetrator from the target could be measured.

In addition the use of a heater may generate convection in the porous target and give an erroneous measurement for the thermal diffusivity. To help investigate this effect the temperature of the target was measured at different locations when the penetrator's heater was active. Preliminary calibration and validation related to the penetrator fitted with the thermal sensor can be found in Paton (2005) where the measurement uncertainty of the heater temperature was calculated to be $0.07 \mathrm{~K}$.

The custom built drop rig, shown in Fig. 7, was designed to simulate a penetrator (or penetrometer) impact on an extraterrestrial surface where, typically, the acceleration due to gravity is lower than on Earth. To simulate the acceleration due to gravity, during the impact, a system of balancing weights are used. Weights at the back and front of the rig are connected by a chain passing over cogs. By keeping the total mass of the weights constant but changing the ratio of their masses their acceleration can be controlled. To accelerate the system quickly up to a desired speed extra weights are added to the back weights. The cable connecting the back weights pass over pulleys at the top of the rig. In this way they provide an upward force on the back weights. When the accelerating weights are released they fall down accelerating the back of the rig upwards and accelerate the front weights, and the penetrator, downwards towards the target. When the accelerating weights hit the ground they are disengaged from the system so the impact dynamics are controlled by the re-

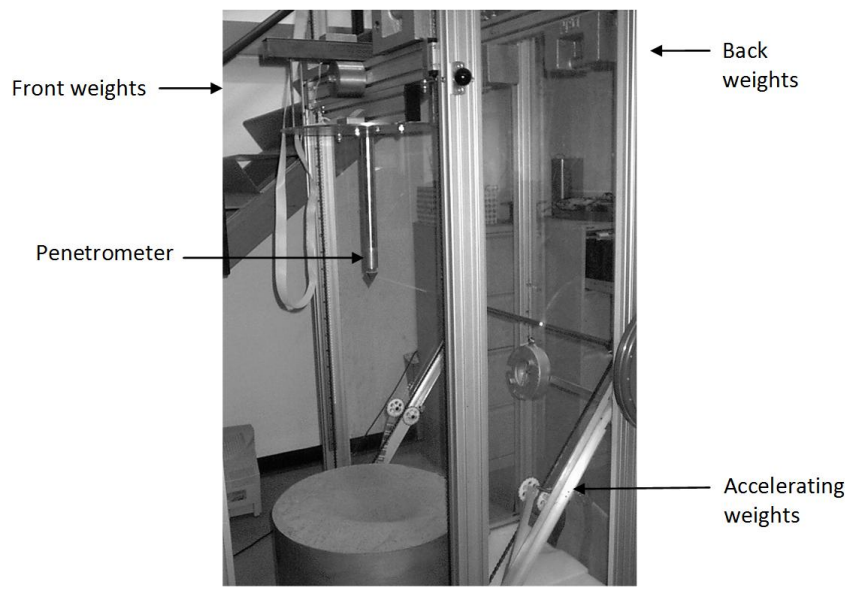

Fig. 7. Penetrometer Test Rig used to suspend and lower a penetrometer (or in our case a penetrator) in a controlled manner using a shaft encoder to measure distance and velocity. The container used for the thermal experiments was actually a smaller bucket than in the image and can be seen in Figs. 9 and 10.

maining balancing weights and the properties of the target. In our case the front and back weights were equalised so there would be no compression of the target, due to the weight of the penetrator pressing down on the target, after the impact.

The targets were prepared by pouring material from a constant height above the top of the bucket. Knowing the mass of the material beforehand and measuring the height of the surface of the poured material yielded a mean density. The surface was generally a bit bumpy making the measurement of its height difficult. To solve this problem the target preparation was finished off by redistributing the top layer of material by hand to make the surface level. The height of the surface was then determined as follows. A ruler was used to measure the vertical distance of the surface below the rim of the container at four locations. The average distance was calculated and then subtracted from the height of the container. Small vertical variations due to hand movements were determined to have a negligible effect on the packing density of the target. This was investigated by measuring and comparing the bulk density of targets prepared with material poured from two different heights.

To investigate the effects of the penetrator impact on the thermal properties of the target, measurements were made in two different ways, as shown in Fig. 8. Firstly a so-called loose target was prepared by pouring the material around the penetrator and then the thermal measurements were made. Secondly the target was prepared without the penetrator and then, after the impact, the thermal measurements were made. The thermal measurements from the loose and impacted targets could then be compared to investigate how the impact modifies the thermal properties of the material. The temperatures from the heater and thermistors, shown in Fig. 9, were read continuously for one hour. The temperature of the heater 


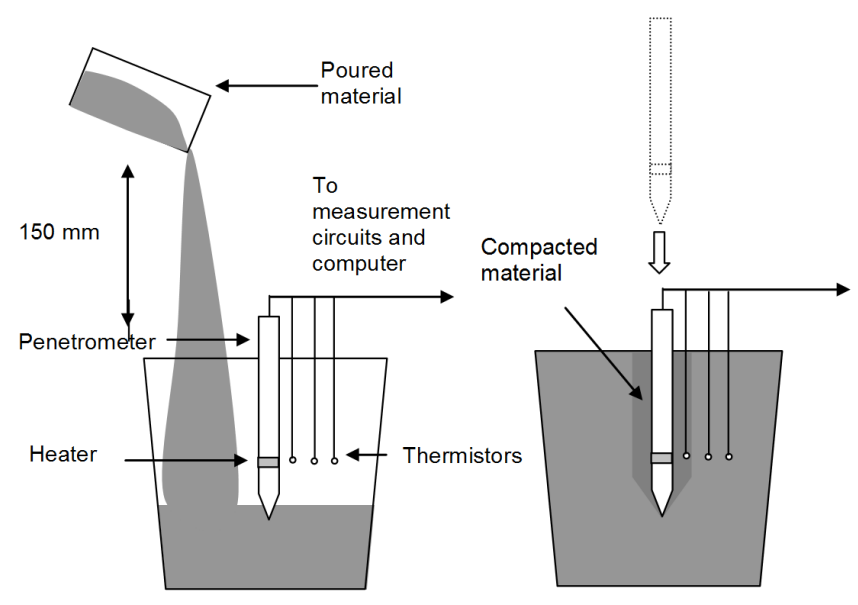

Fig. 8. Experiment preparation to investigate mechanical and thermal effects on porous materials using a penetrator fitted with a sensor to make thermal measurements. On the left is a diagram of an experiment to measure the thermal diffusivity of undisturbed material. On the right is a diagram showing an impacting penetrator and the formation of a possible compaction zone that may give erroneous measurements of the true thermal properties of the bulk material.

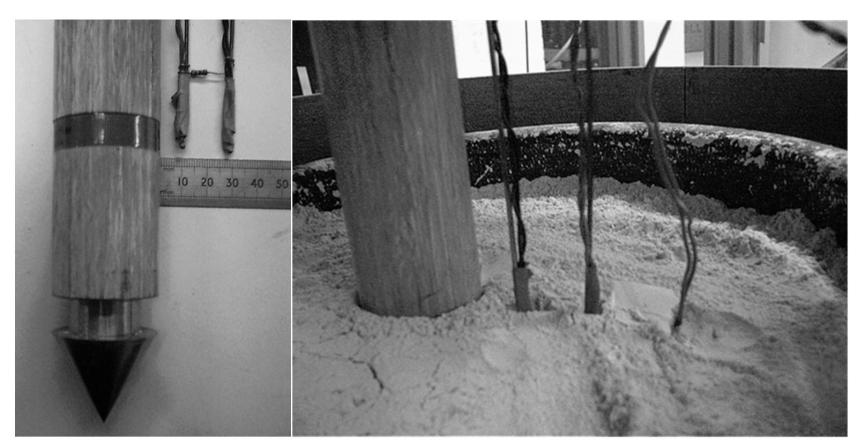

Fig. 9. Position of the thermistors relative to the heater. On the left the thermistors are shown next to the penetrator as they would appear if the target was transparent. On the right is the penetrator and thermistors deployed in the material.

was measured during heating by a circuit using the heater as one resistance in a bridge. The signal was amplified and read into a computer using an ADC11 10 bit analogue to digital converter. Details of the electronics and data logging can be found in Paton (2005). The thermistors were placed at distances of $1 \mathrm{~cm}, 3 \mathrm{~cm}$ and $5 \mathrm{~cm}$ from the foil heater to measure the radial temperature gradient and explore the effects of convection in the target. The thermistors were pushed into the target, each mounted on the end of a rigid piece of thick metal wire, to a level equal to the known heater level in the target.

Planetary regolith analogues, used in the experiments, are shown in Fig. 10. These materials were chosen to reproduce properties of some typical planetary regoliths in terms
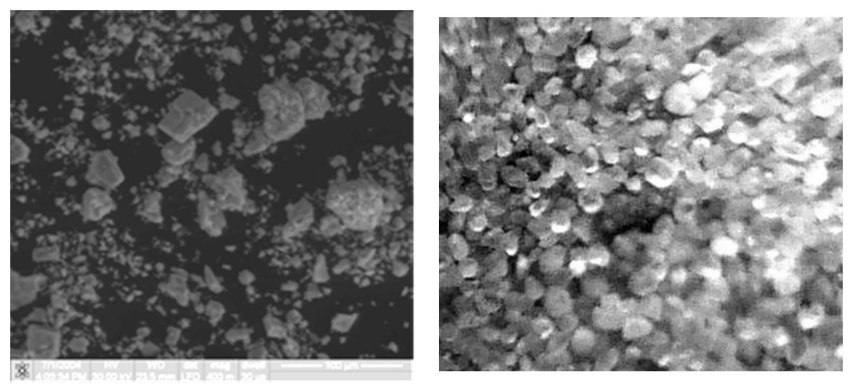

Fig. 10. Left: SEM of limestone powder obtained by R. Greenwood at PSSRI. Right: image of sand grains was obtained by the author using a small camera housed inside a transparent cylindrical tube and immersed in the target. See Paton (2005) for further details.

of their composition and bulk density. They were also chosen with contrasting particle properties to investigate the relation of their thermal properties to properties such as particle size and shape. Limestone powder was selected as it has small, poorly sorted, angular particles contrasting to sand which has large spherical well sorted particles. Sand is a rather general term and geologically this sand would be described as medium grained sand. Close up images of the particles that make up these materials are shown in below in Fig. 10. The images were used to characterise the particles in the materials. The properties of the particles in the target materials are listed in Table 2.

\section{Results and discussion}

Figures 11 and 12 show temperature measurements made in limestone powder and sand over one hour. The materials can easily be distinguished from each other by their temperature profiles. The heater temperature, in limestone powder, rises considerably higher than when placed in sand. This result can be explained in terms of the material microstructure and grain properties. Limestone powder has a higher porosity than sand which means the material is not so tightly packed as the sand grains and has less solid material, per unit volume, to conduct the heat. In addition the grains in the limestone powder are more angular in shape than the sand grains which means the flow of heat is also restricted due to the low Hertz factor (see Sect. 3.1 for the definition).

For both materials the temperature difference profiles of the heater in Figs. 11 and 12 can be split into roughly two sections. From zero to $15 \mathrm{~min}$ there is a steep rise in temperature then after this the temperature difference begins to level off. It is interesting to note that the temperature in the second part appears to fluctuate over short time periods. The amplitude of the fluctuations $(0.2-0.3 \mathrm{~K})$ is close to the measurement error $(0.1 \mathrm{~K})$ and so may be due to measurement set-up. However the onset of the fluctuations appears to be 

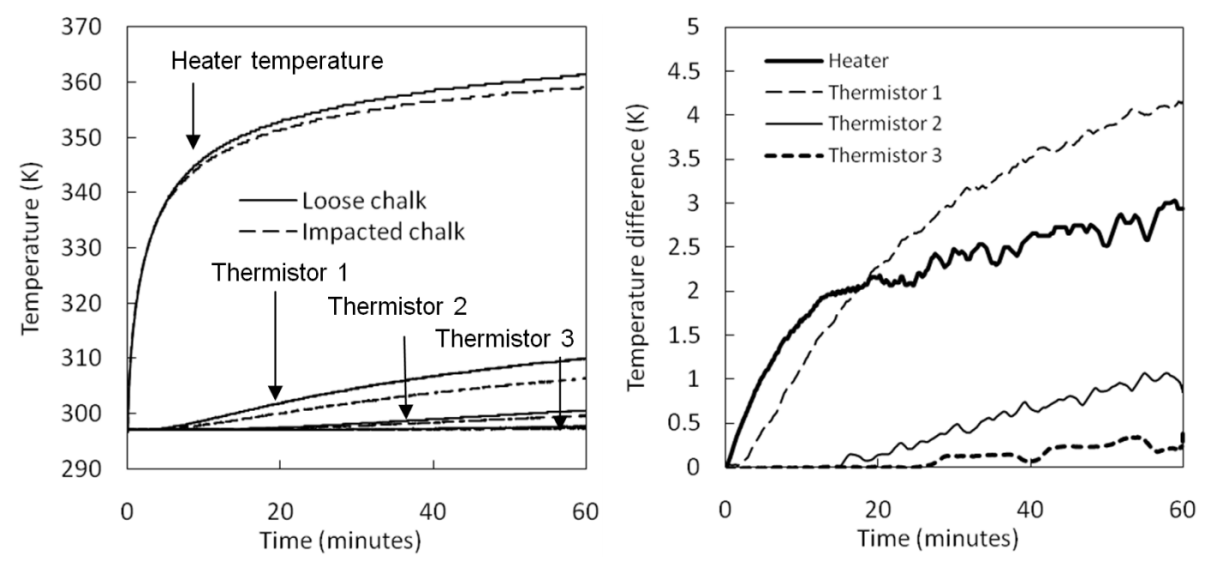

Fig. 11. Laboratory measurements of temperature in limestone powder (left). On the right is the difference in temperature between loose limestone and impacted limestone from the heater and the 3 thermistors. The measurement error on the temperature (left) is $0.07 \mathrm{~K}$ (Paton, 2005 ) and the combined measurement error on the temperature difference (right) is $0.1 \mathrm{~K}$.
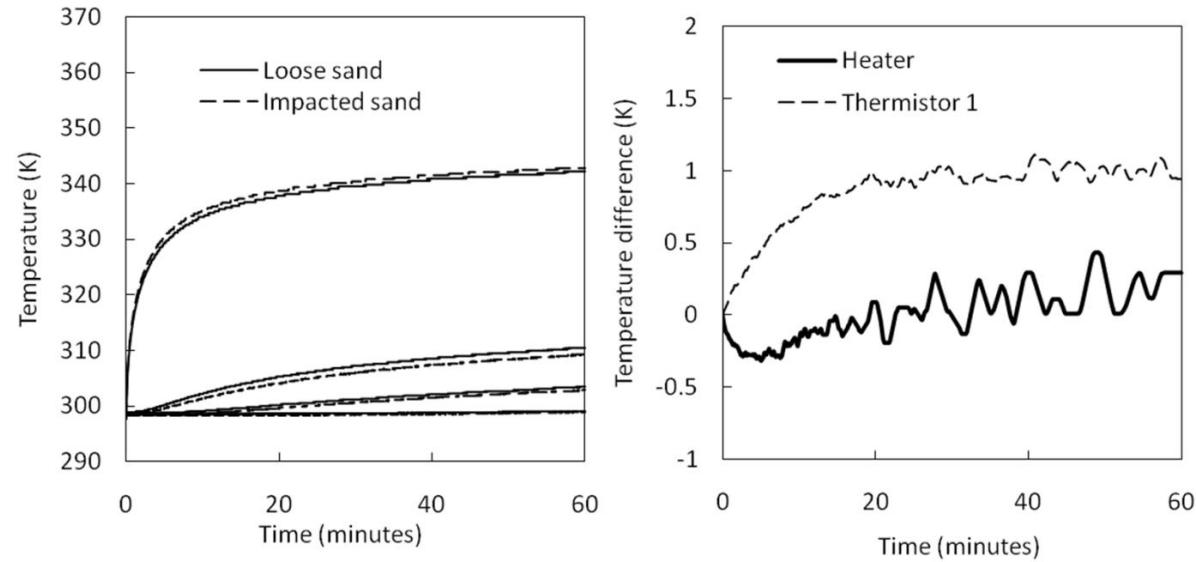

Fig. 12. Laboratory measurements of temperature in sand (left). On the right is the difference in temperature between loose sand and impacted sand from the heater and thermistor 1 . The measurement error on the temperature (left) is $0.07 \mathrm{~K}$ (Paton, 2005) and the combined measurement error on the temperature difference (right) is $0.1 \mathrm{~K}$.

sudden and there may be an unknown physical phenomena at play connected to the heating up of the heater-sensor.

The temperatures measured by the heater and the thermistors, in impacted limestone, are all lower than those temperatures obtained in loose limestone. This is then consistent with a zone of compacted material around the penetrator. A compaction zone would transfer heat more quickly than the undisturbed material because it will have a higher bulk density and the grains will have a greater surface area in contact with each other. In contrast to the result for limestone powder the heater temperature in impacted sand is lower than in loose sand. This suggests the sand is becoming less densely compacted after impact. The difference of temperature is close to the measurement error but at greatest difference in temperature $(\sim 0.5 \mathrm{~K})$ this is substantially larger than the measurement error $(0.1 \mathrm{~K})$.
It is well known (e.g. see Vaid et al., 1981) that under shearing forces, a loosely packed sample will increase its packing density while a densely packed sample may first contract but then have to expand to allow the material to flow. The porosity for random close packed monodisperse spheres is 0.39 when the sample is poured gently into a container (Scott and Kilgour, 1969). The porosity for the jammed state for spheres, where the material cannot be compacted anymore, is 0.36 which is very close to the value for a sample prepared by pouring. In comparison naturally produced granular material can be compacted to lower porosities because they have a large range of particle sizes with the smaller particles filling in the spaces between the large particles.

The particles in our sand sample are close to spherical in shape and contain a small range of particles sizes. It is possible that even though the porosity is initially high $(0.4)$ the sand may quickly reach a jammed state where it has to dilate 


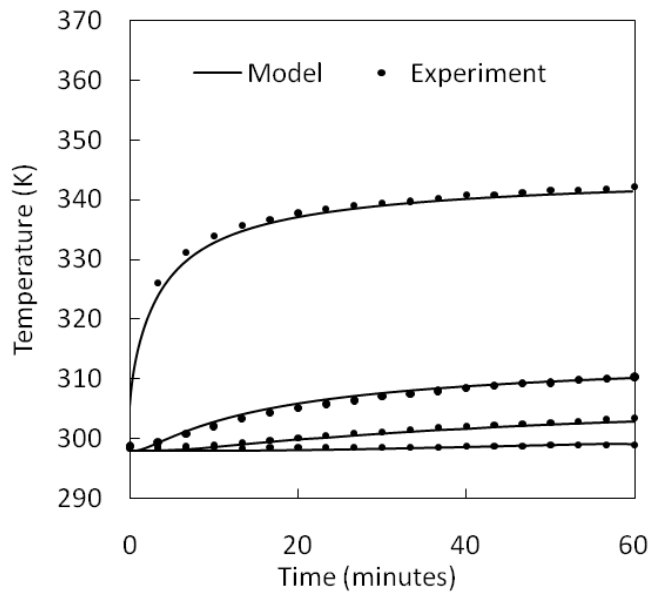

Fig. 13. Model and laboratory measurements in sand compared after moving the model thermistor 1 from 11 to $12.5 \mathrm{~mm}$ from the heater and moving thermistor 2 from 28 to $26.5 \mathrm{~mm}$. The thermal conductivity used for modelling sand was $0.22 \mathrm{~W} \mathrm{~m}^{1} \mathrm{~K}^{-1}$.

in order to allow the penetrator to pass. The lower temperature of the heater in impacted sand may then be a result of a more loosely compacted sample than before the impact.

Alternatively the lower temperature of the impacted sand may due thermal resistance effects at the contact point between the heater and the target. This could be a conceivable problem in highly cohesive granular material when the penetrator bounces or wobbles as it comes to rest and opens the cavity reducing the pressure on its surface, and hence the number of grains contacting the heater. Our experimental set-up was designed so once the penetrator came to rest the force due to gravity would be balanced out to minimise the possibility of movement after reaching its maximum penetration depth. Also the sand we used consisted of very spherical grains which allows the easy flow of the material and no water or cohesive agent was added to the target.

The viscosity and permeability are parameters in the model that can be varied to asses the influence of the role of convection. These were kept constant. The viscosity of air is well known and a value of $2 \times 10^{-5} \mathrm{~kg} \mathrm{~m}^{-1} \mathrm{~s}^{-1}$ was assumed. The reason for keeping the viscosity of air constant was that it is well known air varies by only a few percent over the temperature range in our experiments. The permeability of sand can vary over a wide range depending on the particle size and how well sorted it is. A permeability of $100 \mu \mathrm{m}^{2}$ was assumed for medium grained sand (Bartley and Ruth, 2001). It was noticed increasing the permeability in the convection-diffusion model tends to create better fits with the thermistors.

Figure 13 shows a fit between laboratory measurements in non-compacted sand compared with output from the model. To obtain this fit first the model heater temperature was fitted to the heater temperature in the experiment by varying only the thermal diffusivity in the model and not the con-

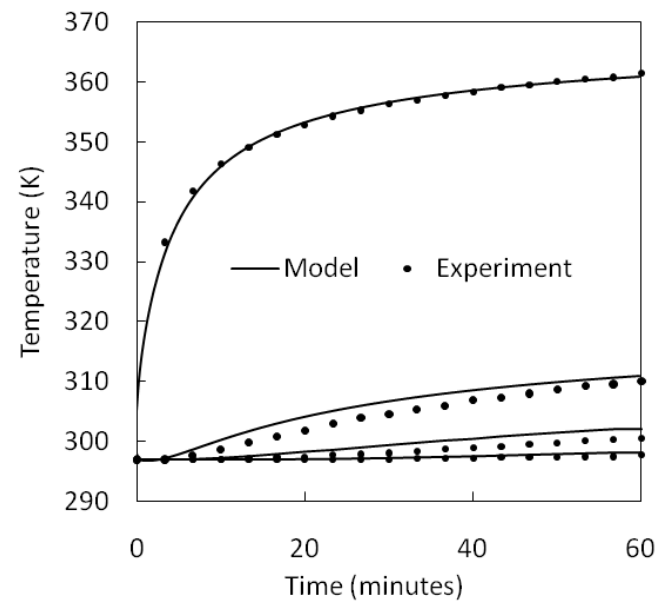

Fig. 14. Model and laboratory measurements in limestone compared after moving the model thermistor 1 from 11 to $14.0 \mathrm{~mm}$ from the heater and moving thermistor 2 from 28 to $31 \mathrm{~mm}$. The thermal conductivity used for modelling sand was $0.16 \mathrm{~W} \mathrm{~m}^{1} \mathrm{~K}^{-1}$.

vective parameters, viscosity and permeability, as these were assumed to be constant. To fit the model to the thermistor measurements a small adjustment had to be made to the location of the thermistors in the model. It was known that the thermistors 1 and 2 were originally $18 \pm 1 \mathrm{~mm}$ away from each other. Pouring sand into the container may have caused some movement of the thermistors towards each other. This was because the thermistors were attached to each other by a metallic rod close to the lower end of the thermistor supporting structure. Material that was poured into the container from above, and mostly from the outer perimeter, was likely to apply some pressure moving the two thermistors towards each other.

Thermistor 1 in the model was moved a distance of $1 \mathrm{~mm}$ further away from the heater and thermistor 2 was moved a distance of $1 \mathrm{~mm}$ closer to the penetrator. The resulting distance between the thermistors in the model is then $16 \mathrm{~mm}$ and the distance of thermistor 1 from the heater was $11 \mathrm{~mm}$. Figure 14 shows a fit between laboratory measurements in limestone compared with output from the model. To fit the model to the measurements required taking output from the model $3 \mathrm{~mm}$ further away from the heater than was the assumed location of the thermistor in the laboratory target. The second thermistor did not fit the model when model output was obtained from the assumed location.

The thermistor measurements could have been influenced by factors not accounted for in the model such as the formation of a compaction zone. To help understand the results more thoroughly, and to compare these results to previous results from Paton (2005), a sensitivity study was made, shown in Fig. 15, of the dependance of the temperature change on knowing the location of the thermistor. The percentage uncertainty is measured on the change in temperature of the material from its initial temperature and not on the the absolute 
Table 2. Analogue planetary regolith properties where $e_{\min }$ is the void ratio of the soil in its densest state, $e_{\max }$ is the void ratio of the soil in its loosest state, $\theta$ is the angle of repose, $\Psi$ is the sphericity, $\rho_{\mathrm{g}}$, is the bulk density of the material in granular form, $\phi$ is the porosity and $\sigma$ is the volumetric strength. The uncertainty on the void ratio, angle of repose, strength and density are due to measurement uncertainty. Details on these properties and their measurement can be found in Paton (2005). The thermal properties are for the solid part of the materials where $k_{\mathrm{S}}$ is the thermal conductivity, $c_{\mathrm{S}}$ is the heat capacity and $\rho_{\mathrm{S}}$ is the density (Bloomfield et al., 1995).

\begin{tabular}{llcc}
\hline Property & Description & Limestone & Sand \\
\hline$d(\mu \mathrm{m})$ & mean particle diameter & 3 & 100 \\
$e_{\min }$ & void ratio of the soil in its densest state & $0.56 \pm 0.02$ & $0.41 \pm 0.01$ \\
$e_{\max }$ & void ratio of the soil in its loosest state & $0.32 \pm 0.01$ & $0.31 \pm 0.01$ \\
$\theta_{\mathrm{r}}$ & angle of repose & $50 \pm 1^{\circ}$ & $28 \pm 1^{\circ}$ \\
$\Psi$ & sphericity & $0.86 \pm 0.11$ & $0.95 \pm 0.10$ \\
$R$ & roughness (0 to 1: angular to rounded) & $0.25 \pm 0.03$ & $0.55 \pm 0.01$ \\
$\rho_{\mathrm{g}}\left(\mathrm{g} \mathrm{cm}^{-3}\right)$ & bulk density of the material in granular form & $1.03 \pm 0.02$ & $1.38 \pm 0.03$ \\
$\phi$ & porosity & $0.60 \pm 0.02$ & $0.40 \pm 0.02$ \\
$\sigma\left(\mathrm{kPa}^{\circ}\right.$ & volumetric strength & $11 \pm 1$ & $170 \pm 12$ \\
$k_{\mathrm{S}}\left(\mathrm{W} \mathrm{m}^{-2}\right)$ & thermal conductivity of solid material & 2.0 & 2.0 \\
$c_{\mathrm{S}}\left(\mathrm{J} \mathrm{kg}^{-1}\right)$ & heat capacity of solid material & 800 & 850 \\
$\rho_{\mathrm{S}}\left(\mathrm{g} \mathrm{cm}^{-3}\right)$ & density of solid material & 2600 & 2700 \\
\hline
\end{tabular}

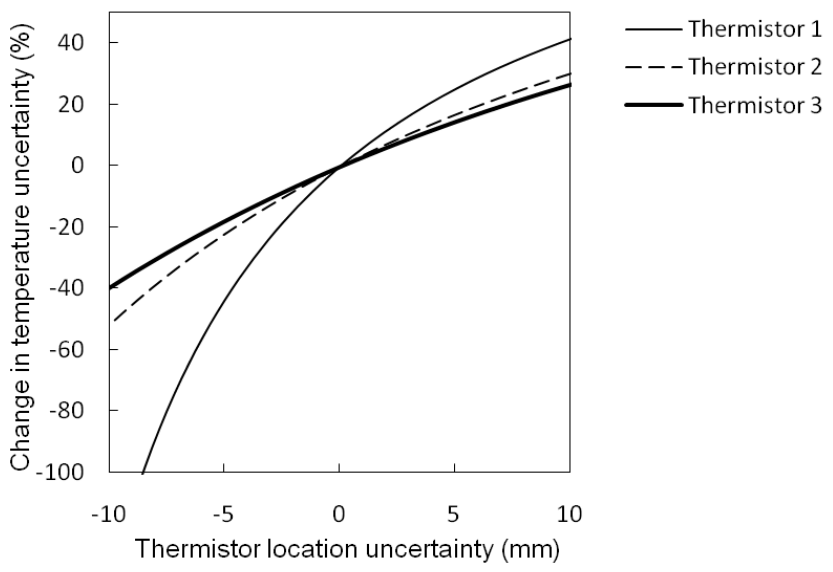

Fig. 15. The temperature change uncertainty relative to knowledge of the location of the thermistors. The percentage is calculated from considering the difference in temperature between the modelled thermistor and the ambient temperature and not from the absolute temperature. It has been calculated for one hour after the heater has been switched on.

temperature i.e. for thermistor 1 where $T_{x=1}$ would be the temperature at the location of thermistor 1 assuming that it had been deployed properly at a radial distance of $10 \mathrm{~mm}$ from the heater (at a depth of $60 \mathrm{~mm}$ ). The temperature, $T_{x}$ is then the temperature taken from a horizontal cross-section running from the heater. All these temperatures were obtained from the temperature map shown in Fig. 6. It can be seen from Fig. 15 that the location of the thermistors need to be accurately known. For example a $2 \mathrm{~mm}$ uncertainty on knowing the position of thermistor 1 , relative to the heater, will result in a $10 \%$ uncertainty on knowing the temperature change. This could then lead to misinterpretation of the results.

The temperature change of thermistor 1 , after one hour in impacted limestone powder, is about two thirds of the temperature change of thermistor 1 in loose limestone powder. This could be interpreted as a compaction zone, created during the impact, transferring the heat away more quickly than in loose limestone powder which does not have a compaction zone. However comparing the temperature changes of the heater, in impacted and loose limestone powder, there is little difference so this interpretation is probably false. From the sensitivity study results in Fig. 15 it can be seen that if thermistor 1 is moved a distance $4 \mathrm{~mm}$ closer to the heater this will cause an increase of $40 \%$ in the temperature change measured compared to the temperature change measured at the original location. Moving thermistor 1 a distance $4 \mathrm{~mm}$ in the other direction will decrease the temperature change by about $15 \%$. It is then likely that thermistor 1 , in impacted limestone powder is closer to the heater than thermistor 1 in loose limestone. The temperature sensors may have moved closer to the centre as were inserted after the impact. As they are pushed in the thermistor rods were kept at a constant distance from the shaft as they entered the target. However the rods were not completely stiff and the thermisters could have been deflected one way or another as they penetrated the target.

The diffusion-convection model developed here, even with the uncertainty regarding the thermistor locations, produces an improvement over a diffusion-only thermal model which suggested much larger temperatures at the locations of the thermistors (Paton, 2005). For sand the temperature change after one hour of heating, for example of thermistor 1, predicted by a diffusion-only thermal model, is around $100 \%$ 
Table 3. Derived thermal diffusivities from fits of models to laboratory measurements. Diffusivity A is derived from a diffusion-only model. Diffusivity B simulates heat transfer by diffusion and convection. The uncertainty in knowing the thermal diffusivity is a result of uncertainties in the density. The diffusivity measurement measured here of $2.1 \mathrm{~m}^{2} \mathrm{~s}^{-1}$ are consistent with thermal conductivity measurements made using other methods. For example the thermal diffusivity is calculated to be $1.8 \mathrm{~m}^{2} \mathrm{~s}^{-1}$ from measured conductivity and density (Vlodeck et al., 2009; Baker and Goodrich, 1984) and assuming a heat capacity of $800 \mathrm{~J} \mathrm{~kg}^{-1} \mathrm{~K}^{-1}$.

\begin{tabular}{lcc}
\hline Sample & $\begin{array}{c}\text { Diffusivity A } \\
\left(10^{-7} \mathrm{~m}^{2} \mathrm{~s}^{-1}\right)\end{array}$ & $\begin{array}{c}\text { Diffusivity B } \\
\left(10^{-7} \mathrm{~m}^{2} \mathrm{~s}^{-1}\right)\end{array}$ \\
\hline Loose limestone powder & $1.87 \pm 0.06$ & $1.05 \pm 0.03$ \\
Impacted limestone powder & $1.97 \pm 0.08$ & $1.11 \pm 0.04$ \\
Loose sand & $3.05 \pm 0.13$ & $2.15 \pm 0.14$ \\
Impacted sand & $2.99 \pm 0.12$ & $2.08 \pm 0.11$ \\
\hline
\end{tabular}

bigger than the temperature change actually measured by thermistor 1 . This would require moving the thermistor virtually, in the model, to only a couple of milimetres from the heater which is difficult to reconcile with the experimental set-up. Similarly the change in temperature after one hour of heating, predicted by a diffusion-only model for thermistor 2 , is around $300 \%$ larger than that measured by thermistor 2 .

Table 3 lists values for diffusivity calculated using a diffusion-only model and a diffusion-convection model. As one would expect the thermal diffusivity of the diffusiononly model is higher than for the diffusion-convection model because a greater amount of heat needs to be carried away by diffusion for the simulated temperatures to fit the measured temperature. The thermal conductivity is normally measured in geophysical applications as it is strongly dependent on water content. Typical values for conduction and bulk density for dry sand are around $0.25 \mathrm{~W} \mathrm{~m}^{-1} \mathrm{~K}^{-1}$ and $1.7 \mathrm{~kg} \mathrm{~m}^{-3}$, respectively (Vlodeck et al., 2009; Baker and Goodrich, 1984). The thermal diffusivity will then be, assuming a heat capacity of $800 \mathrm{~J} \mathrm{~kg}^{-1} \mathrm{~K}^{-1}$, around $1.8 \mathrm{~m}^{2} \mathrm{~s}^{-1}$ which corresponds well with diffusivity derived using a diffusion-convection model. Fine sand, which is close in particle size and has a similar solid thermal conductivity to limestone powder, has a conductivity of around $0.15 \mathrm{~W} \mathrm{~m}^{-1} \mathrm{~K}^{-1}$. This translates to a thermal diffusivity of $0.9 \mathrm{~W} \mathrm{~m}^{-1} \mathrm{~K}^{-1}$ which also corresponds well to the results.

\section{Concluding remarks}

We introduced a thermal probe to make measurements in a planetary regolith and whose deployment by a spacecraft was simulated in the laboratory. The penetrator measured the temperature and thermal diffusivity in planetary regolith analogue materials (sand and limestone powder). The thermal diffusivity for limestone powder was found to be a factor of three lower than sand which can be explained by restricted heat conduction paths due to the angular nature of the limestone powder grains coupled together with its higher bulk porosity.

Thermistors were placed in the target to measure the temperature gradient radially from the penetrator heater. It appeared that convection played a non-negligible role in the transfer of heat away from the penetrator and into the regolith analogues. The derived diffusivity, using a diffusionconvection model fitted to the heater temperature, was found a close match to measurements made using other methods. When a diffusion-only model was fitted to the heater temperature the match was not as good as when using a diffusionconvection model. A diffusion-convection model also fitted the thermistors measurements better than when using a diffusion-only model. Remaining discrepancies between the thermistor measurements and the diffusion-convection model can be explained by the uncertainty in knowing the location of the thermistors relative to the heater.

To improve the measurement scheme used here we recommend the penetrator shaft is filled with foam with known thermal properties to restrict leakage of heat into the penetrator and to allow for more precise measurements. More thermistors added in the vertical direction are desirable to measure the rate of convective flow and to further validate numerical models and to ultimately investigate the mass transfer processes between gas filled planetary regoliths and the atmosphere. An improved experimental technique is required for determination of the precise locations of the thermistors perhaps with a transparent floor for the container so the location of the thermistors can be observed more accurately.

Acknowledgements. We would like to acknowledge the Academy of Finland for grant \#132825. Mark Paton would like to acknowledge the Royal Astronomical Society for a Hosie Bequest studentship.

Edited by: P. Falkner

\section{References}

Andrade Jr., J. S., Costa, U. M. S., and Almeida, M. P: Inertial effects on fluid flow through disordered porous media, Phys. Rev. Lett., 82, 5249-5252, 1999.

Banaszkiewicz, M., Seweryn, K., and Wawrzaszek, R.: A sensor to perform in-situ thermal conductivity determination of cometary and asteroidal material, Adv. Space Res., 40, 226-237, 2007.

Bartley, J. T. and Ruth, D. W.: Experimental investigation of unsteady-state relative permeability in sand-packs, Society of Core Analysis Conference, 22, 1-14, 2001.

Beck, J. L.: Onset of thermal convection in a vertical porous cylinder with a partly conducting and partly penetrative wall, Transport Porous Med., 86, 229-241, 1972.

Birchley, P. N. W., Daniell, P. M., Zarnecki, J. C., and Parker, D. J.: Laboratory determination of the thermal conductivity of liquid 
methane and ethane, in: Proceedings of the Symposium on Titan, ESA SP-338, 311-314, 1992.

Bloomfield, J. P., Brewerton, L. J., and Allen, D. J.: Regional trends in matrix porosity and dry density of the limestone of England, Q. J. Eng. Geol. Hydrog., 28, 132-142, 1995.

Brinkmann, H. C.: On the permeability of media consisting of closely porous particles, J. App. Sci. Res., A1, 81-86, 1947.

Bullard, E.: The flow of heat through the floor of the Atlantic Ocean, Proc. R. Soc. London A, 222, 408-425, 1954.

Darcy, H.: Les Fontines Publiques de la Ville de Dijion, Dalmont, Paris, 1856.

Fletcher, C. A. J.: Computational Techniques for Fluid Dynamics 1: Fundamental and General Techniques, Springer-Verlag, Berlin, 1988.

Foreheimer, P.: Wasserbewegung durch Boden, Zeits. V. deutsch. Ing., 45, 1782-1788, 1901.

Gori, F. and Corasaniti, S.: Theoretical prediction of the thermal conductivity and temperature variation inside mars soil analogues, Planet. Space Sci., 52, 91-99, 2004.

Harri, A.-M., Linkin, V., Polkko, J., Marov, M., Pommereau, J.P., Lipatov, A., Siili, T., Manuilov, K., Lebedey, V., Lehto, A., Pellinen, R., Pirjola, R., Carpentier, T., Malique, C., Makarov, V., Khloustova, L., Esposito, L., Maki, J., Lawrence, G., and Lystsev, V.: Meteorological observations on Martian surface: met-packages of Mars-96 Small Stations and Penetrators, Planet. Space Sci., 46, 779-793, 1998.

Harri, A.-M., Marsal, O., Lognonne, P., Leppelmeier, G. W., Spohn, T., Glassmeier, K.-H., Angrilli, F., Banerdt, W. B., Barriot, J. P., Bertaux, J.-L., Berthelier, J. J., Calcutt, S., Cerisier, J. C., Crisp, D., Dehant, V., Giardini, D., Jaumann, R., Langevin, Y., Menvielle, M., Musmann, G., Pommereau, J. P., Di Pippo, S., Guerrier, D., Kumpulainen, K., Larsen, S., Mocquet, A., Polkko, J., Runavot, J., Schumacher, W., Siili, T., Simola, J., and Tillman, J. E.: Network science landers for Mars, Adv. Space Res., 23, 1915-1924, 1999.

Harri, A.-M., Makkonen, P., Polkko, J., Lappalainen, H., Pellinen, R., Vorontsov, V., Polyakov, A., Ivankov, A., Linkin, V., Gotlib, V., and Lipatov, A.: METNET - the NextGeneration of Atmospheric Science Missions to Mars, Paper-Nr.: IAC-03-Q.3.b.09, 54th International Astronautical Conference, 29 September-3 October, Bremen, Germany, 2003.

Hagermann, A. and Spohn, T.: A method to invert P/Wirtanen temperature recording for the subsurface temperature of MUPUS, Adv. Space Sci., 23, 1333-1336, 1999.

Hsu, C. T. and Cheng, P.: Thermal dispersion in a porous medium, Int. J. Heat Mass Tran., 33, 1587-1597, 1990.

Hütter, E. S., Kömle, N. I., Kargl, G., and Kaufmann, E.: Determination of the effective thermal conductivity of granular materials under varying pressure conditions, J. Geophys. Res., 113, E12004, doi:10.1029/2008JE003085, 2008.

Incropera, F. P. and Dewitt, D. P.: Fundementals of Heat and Mass Transfer, 5th Edn., John Wiley \& Sons, 2009.

Klinkenberg, L. J.: The permeability of porous media to liquid and gases, API Drilling and Production Practice, American Petroleum Institute, 200-213, 1941.

Kömle, N. I., Kargl, G., and Seiferlin and Marczewski, W.: Measuring thermo-mechanical properties of cometary surfaces: In situ methods, Earth Moon Planets, 90, 269-282, 2002.

Lunne, T., Robertson, P. K., and Powell, J. J. M.: Cone Penetration
Testing in Geotechnical Practice, Blackie Academic and Professional, London, 1997.

Neuman, S. P.: Theoretical derivation of Darcy's law, Acta Mech., 25, 153-170, 1977.

Nield, D. A. and Adrian, B.: Convection in Porous Media, 3rd Edn., Springer, 2006.

Nouri-Borujerdi, A., Noghrehabadi, A. R., and Rees, D. A. S.: Influence of Darcy number on the onset of convection in a porous layer with a uniform heat source, Int. J. Thermal Sci., 47, 1020 1025, 2008.

Patankar, S. V.: Numerical heat transfer and fluid flow, New York, NY, Hemisphere, 1980.

Paton, M. D.: Penetrometry of NEOs and other Solar System objects, Ph.D. thesis, The Open University, UK, 2005.

Paton, M. D., Kargl, G., and Ball, A. J.: Computer modeling of a penetrator thermal sensor, Adv. Space Res., 46, 337-345, 2010.

Pinna, S., Angrilli, F., Kochan, H., and Richter, L.:, Development of the mobile penetrometer (Mole) as sampling tool for the Beagle2 Lander on Mars Express, Adv. Space Res., 28, 1231-1236, 2001.

Presley, M. A. and Christensen, P. R.: Thermal conductivity measurements of particulate materials: 2. Results, J. Geophys. Res., 102, 6551-6566, 1997.

Rowe, R. K.: Basic behaviour and site characterisation, in: Geotechnical and Geoenvironmental Engineering Handbook, edited by: Rowe, R. K., Kluwer Academic Publishers, Massachusetts, 89-91, 2001.

Scott, G. D. and Kilgour, D. M.: The density of random close packing of spheres, Br. J. Appl. Phys. (J. Phys. D), 2, 863-866, 1969.

Scott, K. M. and Pain, C.: Regolith Science, 1st Edn., Springer, 2009.

Smith, P. H., Tamppari, L. K., Arvidson, R. E., Bass, D., Blaney, D., Boynton, W. V., Carswell, A., Catling, D. C., Clark, B. C., Duck, T., Dejong, E., Fisher, D., Goetz, W., Gunnlaugsson, H. P., Hecht, M. H., Hipkin, V., Hoffman, J., Hviid, S. F., Keller, H. U., Kounaves, S. P., Lange, C. F., Lemmon, M. T., Madsen, M. B., Markiewicz, W. J., Marshall, J., McKay, C. P., Mellon, M. T., Ming, D. W., Morris, R. V., Pike, W. T., Renno, N., Staufer, U., Stoker, C., Taylor, P., Whiteway, J. A., and Zent, A. P.: $\mathrm{H}_{2} \mathrm{O}$ at the Phoenix landing site, Science, 325, 58-61, 2009.

Smits, K. M., Sakaki, T., Limsuwat, A., and Illangasekare, T. H.: Determination of the thermal conductivity of sands under varying moisture, drainage/wetting, and porosity conditions - applications in near-surface soil moisture distribution analysis, AGU Hydrology Days 2009, Colorado State University, 2009.

Smrekar, S., Catling, D., Lorenz, R., Magalhaes, J., Moersch, J., Morgan, P., Murphy, J., Murray, B., Presley-Holloway, M., Yen, A., Zent, A., and Blaney, D.: Deep Space 2: The Mars Microprobe Mission, J. Geophys. Res., 104, 27013-27030, 1999.

Spohn, T., Seiferlin, K., Hagermann, A., Knollenberg, J., Ball, A. J., Banaszkiewicz, M., Johannes, J., Gadomski, S., Gregorczyk, W., Grygorczuk, J., Hlond, M., Kargl, G., Kührt, E., Kömle, N., Krasowski, J., Marczewski, W., and Zarnecki, J. C. A.: MUPUS - A thermal and mechanical properties probe for the Rosetta lander Philae, Space Sci. Rev., 128, 339-362, 2007.

Stöcker, J. and Thiel, M.: ROSETTA lander subsystem specification, Work Package 5350: ANCHORING SYSTEM, RO-LASSP-3101, Issue: 3, MPE Garching, 1998.

Svitek, T. and Murray, B.: Winter frost at Viking lander 2 site, J. Geom. Res., 95, 1495-1510, 1990. 
Ulrichs, J. and Campbell, M. J.: Radiative heat transfer in the lunar and Mercurian surfaces, Icarus, 2, 180-188, 1969.

Urquhart, M. L. and Smrekar, S. E.: Estimation of soil thermal conductivity from a Mars Microprobe-type penetrator, 31st Annual Lunar and Planetary Science Conference, 13-17 March, Houston, Texas, abstract no. 1781, 2000.
Vaid, Y., Byrne, P. M., and Hughes, J. M. O.: Dilation angle and liquefaction potential, J. Geotech. Eng., 103, 1003-1008, 1981.

van Gelder, M. F.: A thermistor based method for measurement of thermal conductivity and thermal diffusivity of moist food mateials at high temperatures, Ph.D. thesis, Virginia Polythechnic Institute and State University, 1998. 